\title{
Simultaneous Channel and OSNR Monitoring Using a Polarization-Selective Modulator and an LED
}

\author{
Chun-Liang Yang, San-Liang Lee, Member, IEEE, Hen-Wai Tsao, Member, IEEE, and \\ Jingshown Wu, Senior Member, IEEE
}

\begin{abstract}
A compact module including a polarization-selective electrooptic modulator and a light-emitting diode (LED) sensor is utilized for optical signal-to-noise-ratio (OSNR) monitoring and channel recognition. The modulator acts as a polarizer and provides signal dithering to improve the detection sensitivity, while the LED is used as a channel sensor. The channel sensor can monitor a wide wavelength range that is rapidly adjustable and can have much relaxed tolerance on temperature control. The combination of a polarization controller/rotator and the polarized modulator can accurately measure the OSNR between 5 and $35 \mathrm{~dB}$ with an error less than $0.5 \mathrm{~dB}$.
\end{abstract}

Index Terms-Channel monitoring, fiber networks, optical performance monitoring, optical signal-to-noise-ratio (OSNR) monitoring, wavelength-division multiplexing (WDM).

\section{INTRODUCTION}

W AVELENGTH-division-multiplexed (WDM) networks require performance monitoring of various signal parameters, including power, wavelength, optical signal-to-noise ratio (OSNR), and so on for providing quality of service and assuring network survivability. There have been many approaches proposed for detecting the channel wavelength and the OSNR [1]-[6]. For instance, channel monitoring can be performed with a tunable Fabry-Pérot (FP) filter [1] or an acoustooptic tunable filter [2]. On the other hand, OSNR monitoring schemes were demonstrated by using the polarization extinction between WDM signals and noise [3], [4]. These techniques utilize a combination of a polarization rotator and a linear polarizer to measure the OSNR. The measurements are based on the assumption that the signal is polarized while the noise is not. Different combinations of optical filters or signal processing schemes can also be used [1], [5], [6].

We propose here a novel approach for simultaneous channel and OSNR monitoring. The goal is to realize a compact multiparameter monitoring module that can provide wide dynamic range and flexible operation. The approach uses a light-emitting diode (LED) as a sensor for channel recognition and a polarization-selective electrooptic (EO) modulator. The modulator not only provides a dithering signal for the LED and photodiode to

Manuscript received August 19, 2003; revised October 17, 2003. This work was supported in part by the Computer and Communications Research Laboratory, Industrial Technology Research Institute, Taiwan, under Grant T2-91 034-14 and in part by the Ministry of Education, Taiwan, under Grant 89-E-FA06-2-4.

C.-L. Yang and S.-L. Lee are with the Deptartment of Electronic Engineering, National Taiwan University of Science and Technology, Taipei, Taiwan 106, R.O.C. (e-mail: sanlee@et.ntust.edu.tw).

H.-W. Tsao and J. Wu are with the Deptartment of Electric Engineering, National Taiwan University, Taipei, Taiwan 106, R.O.C.

Digital Object Identifier 10.1109/LPT.2004.823756

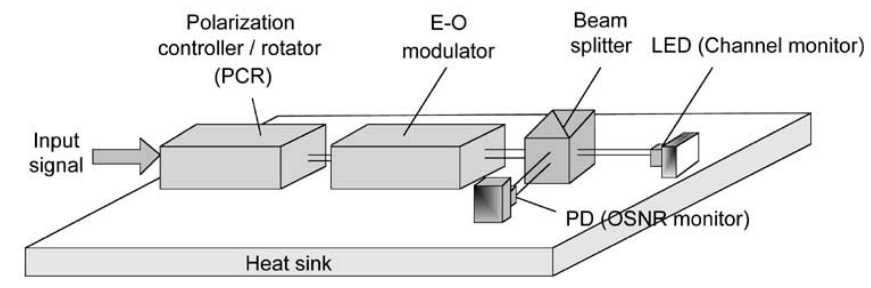

Fig. 1. Schematic diagram of the proposed monitoring module.

improve the detection sensitivity, but also acts as a linear polarizer for measuring the OSNR.

\section{OPERATION PRINCIPLES}

The proposed module is schematically shown in Fig. 1. It can be placed in WDM networks to monitor multiple channels in a scanning mode or a tunable wavelength channel, which may come from a tunable laser or wavelength converter. For monitoring multiple channels, a tunable optical filter (TOF) can be used to scan through the entire wavelength band to select the channels. The OSNR monitoring is performed with a polarization controller/rotator (PCR), a polarized EO modulator, and a photodetector. On the other hand, the channel number is recognized with the optical modulator and an LED. For multichannel monitoring, channel recognition with the LED sensor can alleviate the requirement of a TOF with precise wavelength control and allow for fast channel scanning.

To enable the needed functions, the EO modulator must be very polarization-selective. The modulator can be one with a linear polarizer at its input or one with a polarization-sensitive waveguide structure where one of the polarization is leaky as it propagates in the modulator [7]. With such a modulator and a PCR, the channel OSNR can be measured in a similar way as the polarization nulling approach [4]. At the same time, the modulator applies a dithering signal for the monitoring paths in order to improve the detection sensitivity. The channel wavelength can be detected from the induced junction voltage across the LED sensor. The principle is very similar to the wavelength detection approach that utilizes the wavelength-dependent transparent properties in an FP laser diode (FPLD) [8] or a semiconductor optical amplifier (SOA) [9]. Like an FPLD or SOA, an LED has a gain material of which the transparent current decreases as the wavelength moves to the long-wavelength side. Its junction volatage varies monotonically with the difference between the transparent current and the bias current. Thus, the induced voltage is a function of wavelength.

The LED sensor can provide many advantages over the previously demonstrated channel monitoring approaches. It can 


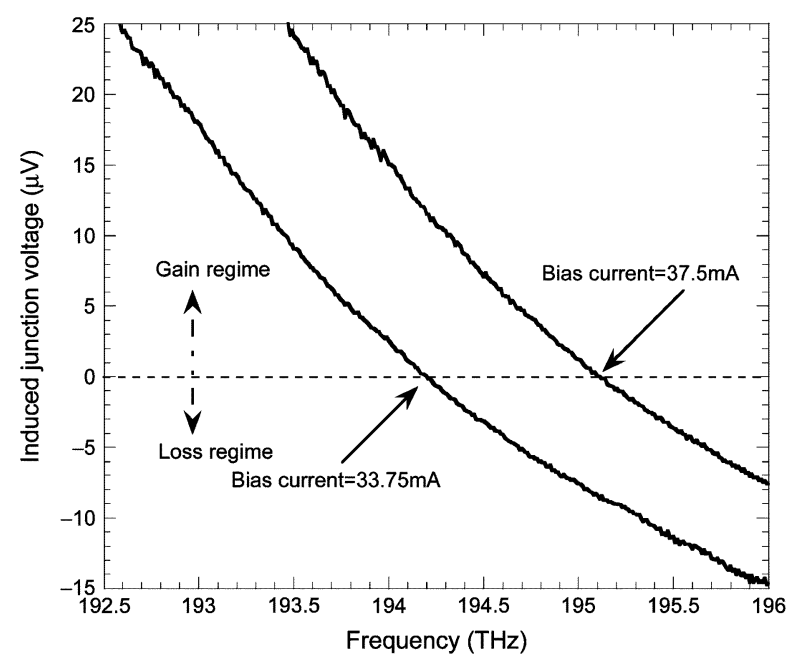

Fig. 2. Induced junction voltage of an LED versus wavelength for different bias current.

monitor a wide range of wavelengths. Its monitoring window can be easily and rapidly tuned to cover different wavelength bands by adjusting its bias current. The induced voltage of an edge-emitting LED is shown in Fig. 2. The induced junction voltage in response to a dithered input light can be detected with a narrow electric bandpass filter to eliminate the noise associated with the detection process. The output signal is very sensitive to wavelength change. Using a lock-in amplifier to detect the LED voltage allows covering a larger wavelength range because the voltages for wavelengths residing in the gain regime and loss regime can be distinguished. When only a few channels are to be monitored, simple amplifier and filtering circuits without using the phase-sensitive detection can be used. The critical concern for using an LED sensor is that it may require critical temperature control. Since the LED needs to simply distinguish the channels from each other rather than to accurately detect the channel wavelength, the detection window is relatively large, so the required temperature control can be relaxed.

For OSNR monitoring, the PCR switches the polarization states to where the maximal or minimal value occurs at the $\mathrm{EO}$ modulator output. The maximal voltage measured by the photodiode indicates the signal power plus half of the noise power, while the minimal voltage corresponds to half of the noise power if the signal is completely suppressed by the polarized modulator. Thus, the OSNR can be calculated from the two extreme voltage values. The channel power can also be obtained from these values. The channel monitoring is performed when the polarization is rotated to give the maximal voltage. The modulator also applied a single-tone sinusoidal signal to the input signal and noise to improve the detection quality for the channel monitoring path.

\section{EXPERIMENTS}

The key components of our approach is the EO modulator and LED sensor. In order for demonstrating the feasibility, we choose a JDSU-made bias-free amplitude modulator that is pigtailed with a polarization-maintaining fiber at the input end and a standard single-mode fiber at the output end. The modulator is made of the annealed proton-exchanged waveguide, where only one polarization is guided. Its polarization extinction ratio
TABLE I

INDUCED VOLTAGES AND FLUCTUATIONS OF THE LED SENSOR FOR EIGHT 100-GHz-SPACED CHANNELS

\begin{tabular}{c|c|c|c|c|c|c|c|c}
\hline $\begin{array}{c}\text { Channel } \\
\text { frequency }(\mathbf{T H z})\end{array}$ & $\mathbf{1 9 3 . 9}$ & $\mathbf{1 9 4 . 0}$ & $\mathbf{1 9 4 . 1}$ & $\mathbf{1 9 4 . 2}$ & $\mathbf{1 9 4 . 3}$ & $\mathbf{1 9 4 . 4}$ & $\mathbf{1 9 4 . 5}$ & $\mathbf{1 9 4 . 6}$ \\
\hline $\begin{array}{c}\text { Induced voltage } \\
(\mu \mathbf{V})\end{array}$ & 14.74 & 13.13 & 11.44 & 9.83 & 8.50 & 7.13 & 5.71 & 4.44 \\
\hline $\begin{array}{c}\text { Fluctuation }(\mu \mathbf{V}) \\
\text { for } \Delta \lambda= \pm \mathbf{0 . 0 2} \mathbf{n m}\end{array}$ & 0.18 & 0.22 & 0.05 & 0.08 & 0.12 & 0.18 & 0.12 & 0.1 \\
\hline
\end{tabular}

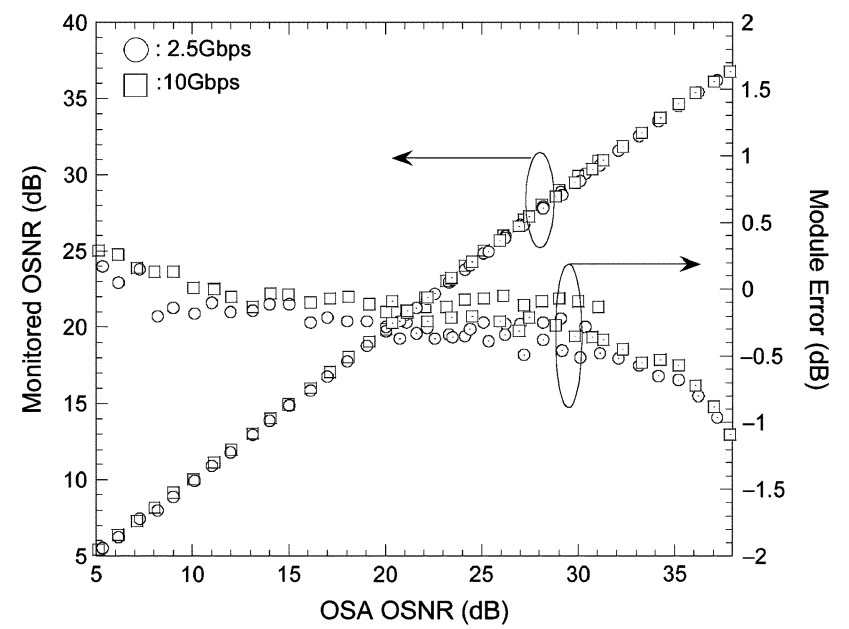

Fig. 3. Measured OSNR values and errors for 2.5- (circles) and 10-Gb/s (squares) bit rates.

(PER) is larger than $47 \mathrm{~dB}$. The LED sensor is a $1.55-\mu \mathrm{m}$ edgeemitting LED of which the induced voltage versus the input wavelength is shown in Fig. 2. The LED is attached to a temperature controlled housing to stabilize its operation temperature. An external-cavity tunable laser is used as the input source and is combined with an amplified spontaneous emission (ASE) noise source. A TOF of 0.265 -nm bandwidth is placed before the monitoring module to simulate the channel selection function. The channel wavelength is then detected by the LED sensor and a lock-in amplifier. The frequency of the dithering signal is $20 \mathrm{kHz}$.

For clarity, the induced voltages for eight $100-\mathrm{GHz}$-spaced dense WDM (DWDM) channels are listed in Table I. Typically, the wavelength locker of a DWDM source can assure the wavelength drift of the channel to be within $\pm 0.02 \mathrm{~nm}$. Within this amount of wavelength drift, the induced voltage between the adjacent channels can be clearly distinguished, as indicated in Table I. Moreover, due to the large separation in the detected voltage between adjacent channels, the tolerance on temperature control of the sensor can be relaxed to $0.24^{\circ} \mathrm{C}$. Though there is a critical concern about the reliability for using such an active component as the channel sensor, we have verified that this type of channel monitor is very reliable and stable after preaging the sensor [10].

Fig. 3 compares the OSNR measured by our module and by a high-performance optical spectrum analyzer (OSA). The measured OSNR was corrected from the factors that are different for the two measurement techniques. The factors include the $3-\mathrm{dB}$ difference in the measured noise level since only half of the ASE noise is measured by the polarized modulator. They also include the difference $(4.2 \mathrm{~dB})$ in the filter bandwidth between the TOF and the monochrometer of the OSA. Due to the limitation of 


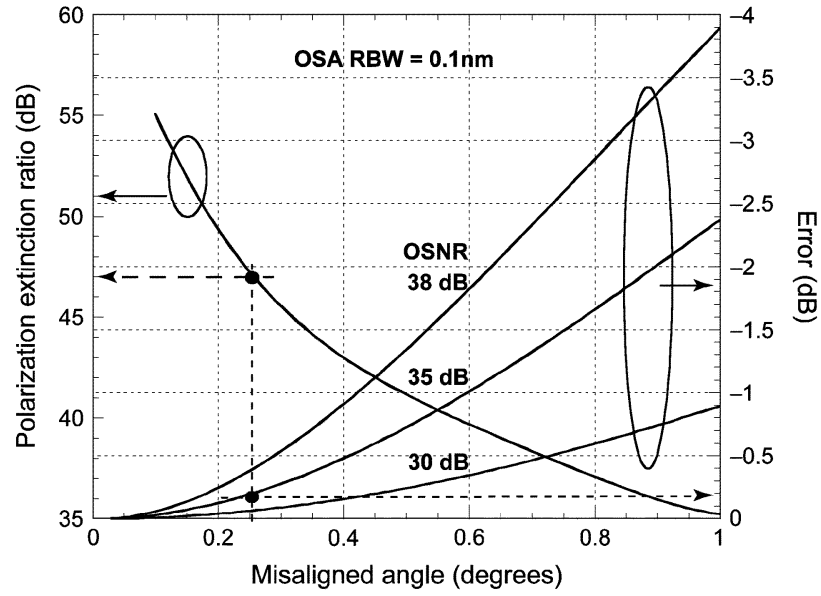

Fig. 4. Simulated results of measurable PER and the corresponding error in OSNR monitoring against the misalignment of polarization controller.

available apparatus in the experiments, we combined the ASE noise source from an erbium-doped fiber amplifier (EDFA) with the signal for measuring OSNR up to $30 \mathrm{~dB}$. For measuring a larger value of OSNR, the signal was directly amplified with the EDFA, i.e., the noise source is cascaded in series with the signal source. The results indicate that the measured OSNR using the proposed module matches very well with the data measured by the OSA.

The module can measure the OSNR between 5 and $35 \mathrm{~dB}$ while the error is kept within $0.5 \mathrm{~dB}$. The error increases dramatically for measuring an OSNR larger than $35 \mathrm{~dB}$. This is mainly due to the fact that the signal power is not completely suppressed in measuring the noise power. When the OSNR is large, the scheme is sensitive to the misalignment between the output polarization state of the PCR and the polarization axis of the modulator. The effect of polarization misalignment was simulated by using the VPItransmissionMaker software and the results are depicted in Fig. 4. Since the modulator's PER can be measured to be around $47 \mathrm{~dB}$ for both $2.5-$ and $10-\mathrm{Gb} / \mathrm{s}$ signals by using the same PCR, the PCR can be adjusted to make the misalignment smaller than $0.25^{\circ}$. This degree of misalignment contributes $<0.2 \mathrm{~dB}$ of error in measuring up to $35-\mathrm{dB}$ OSNR. The error increases to $0.4 \mathrm{~dB}$ in measuring a $38-\mathrm{dB}$ OSNR for the same misalignment. Therefore, the PCR needs to have better precision in polarization adjustment so as to measure a larger OSNR.

Another factor that might contribute to the OSNR error is the polarization-mode dispersion (PMD) caused by the EDFA when the signal is directly amplified. The PMD value for cascading the EDFA (with a built-in isolator) with the TOF was measured to be about $1.26 \mathrm{ps}$. With the influence of PMD, it is difficult to completely suppress the signal during noise measurement. This effect may contribute to the difference in the measured OSNR $(0.168 \mathrm{~dB})$ between the cases that the ASE source is added in parallel with the signal path or cascaded in series. The effect of PMD is also more severe for monitoring a higher OSNR. The influence of PMD on the measured OSNR needs further investigation.

Fig. 5 shows the stability of the channel and OSNR monitoring over $2 \mathrm{~h}$. It was measured without dedicated isolation of the module from fluctuation of ambient conditions. The results indicate good stability for both monitoring paths.

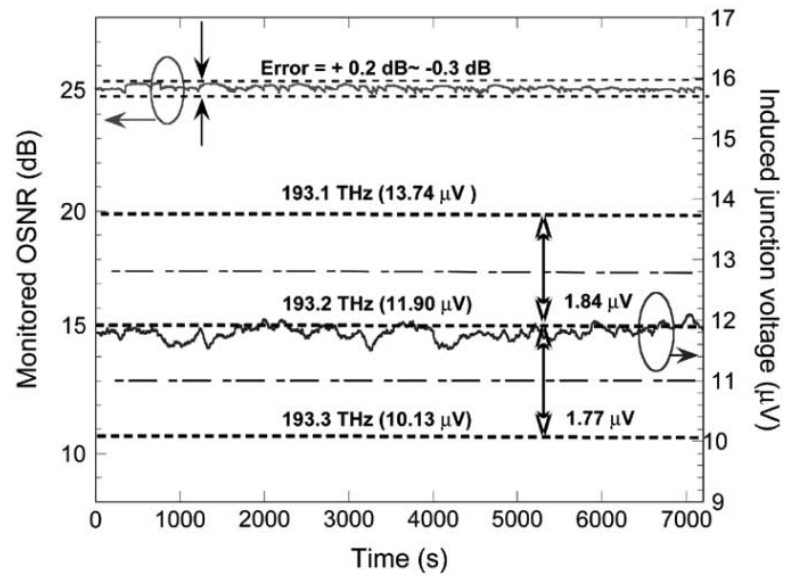

Fig. 5. Stability of channel and OSNR monitoring over $2 \mathrm{~h}$ of continuous measurement. The OSNR was set at $25 \mathrm{~dB}$ during the measurement.

\section{SUMMARY}

We have demonstrated simultaneous channel and OSNR monitoring by using an LED and a polarization-selective EO modulator. The two key components and the photodiode can be packaged into a compact module. The channel monitoring can cover a wide wavelength range and is tunable; and the OSNR monitoring can be accurate over a wide dynamic range.

\section{REFERENCES}

[1] K.-U. Chu, K.-Y. Park, S. B. Lee, C.-H. Lee, S.-Y. Shin, and Y. J. Oh, "A simple and low-cost optical channel analyzer for a dense wavelengthdivision multiplexing system," in Optoelectronics and Communications Conf. (OECC 2000) Tech. Dig., July 2000, pp. 474-475.

[2] Q. Li, A. A. Au, C.-H. Lin, I. V. Tomov, and H. P. Lee, "Performance characteristics of a WDM channel monitor based on an all-fiber AOTF with an on-fiber photodetector," IEEE Photon. Technol. Lett., vol. 15, pp. 718-720, May 2003.

[3] M. Rasztovits-Wiech, M. Danner, and W. R. Leeb, "Optical signal-tonoise ratio measurement in WDM networks using polarization extinction," in Proc. Eur. Conf. Optical Communications (ECOC'98), Sept. 1998, pp. 549-550.

[4] J. H. Lee, D. K. Jung, C. H. Kim, and Y. C. Chung, "OSNR monitoring technique using polarization-nulling method," IEEE Photon. Technol. Lett., vol. 13, pp. 88-90, Jan. 2001.

[5] Data Sheet of Agilent Optical Spectrum Analyzer WDM Test Application Product Overview. Educator's Corner-Resource Guide for Engineering Educators. Agilent Technologies. [Online] Available: http://www.educatorscorner.com/index.cgi?CONTENT_ID= 2647LW_Optical_Spectrum_Analyzer.pdf

[6] W. Chen, S. Zhong, Z. Zhu, W. Chen, and Y.-J. Chen, "Adding OSNR monitoring functionality on AWG based power monitoring circuits," in Proc. Optical Fiber Communications (OFC 2002), Mar. 2002, pp. 668-670.

[7] E. L. Wooten, K. M. Kissa, A. Yi-Yan, E. J. Murphy, D. A. Lafaw, P. F. Hallemeier, D. Maack, D. V. Attanasio, D. J. Fritz, G. J. McBrien, and D. E. Bossi, "A review of lithium niobate modulators for fiber-optic communications systems," IEEE J. Select. Topics Quantum Electron., vol. 6, pp. 69-80, Jan./Feb. 2000.

[8] S.-L. Lee, Y.-Y. Hsu, and C.-T. Pien, "High-resolution wavelength monitoring using differential/ratio detection of junction voltage across a diode laser," IEEE Photon. Technol. Lett., vol. 13, pp. 872-874, Aug. 2001.

[9] S.-L. Lee, "Wavelength measurement and tracking using semiconductor laser amplifiers for applications in photonic networks," IEEE Photon. Technol. Lett., vol. 10, pp. 439-441, Mar. 1998.

[10] S.-L. Lee and Y.-Y. Hsu, "Study of effect of aging on transparent current stability of semiconductor lasers," Electron. Lett., vol. 37, pp. 767-769, June 2001. 